\title{
Large Thin Area Preparation of Cross-Sectional TEM Specimens of III-V Semiconductors
}

\author{
Jian-Guo Zheng* \\ *Materials Characterization, Laboratory for Electron and X-ray Instrumentation, Calit2, \\ University of California, Irvine, CA 92697-2800
}

Defects and interfaces in III-V semiconductors and related superlattices affect their physical properties. Transmission electron microscopy (TEM) is a powerful and unique tool to study these defects and interfaces, but TEM experiments require electron transparent thin specimens. Preparing large thin area of TEM specimens is a challenge for most of microscopists. My experiments show that it is possible to meet this challenge by modifying conventional cross-sectional TEM specimen preparation method or using tripod-related polishing method. This paper reports some typical examples.

Cross-sectional TEM specimen of $17 \mu \mathrm{m}$ thick polar GaN layer grown on (0001) sapphire substrate was successfully prepared (Fig. 1a). Various defects such as thread dislocations (Fig. 1b) and stacking faults (Fig. 1c) can be clearly imaged from the layer surface to the substrate under different diffraction conditions. The decreasing of thread dislocation density with layer thickness can be seen in the $20 \mu \mathrm{m}$ thick semi-polar GaN layer grown on m-plane sapphire substrate (Fig. 2). The nature of large growth defects with a diameter of $30 \mu \mathrm{m}$ in GaN/AIN superlattices was determined in a crosssectional TEM specimen with $200 \mu \mathrm{m}$ wide thin area. Part of the thin area of about $15 \mu \mathrm{m}$ wide is shown in Fig. 3, where one TEM specimen contains two relatively rotated pieces of the GaN/AlN superlattices. Cross-sectional STEM images with atomic scale resolution was achieved in GaInAs/GaAlAs superlattices which is about 4-5 $\mu \mathrm{m}$ away from a device surface (Fig. 4).

Conventional cross-sectional TEM specimen preparation procedure includes many steps such as cutting, gluing, slicing, mounting, mechanical polishing, dimpling and ion milling. To prepare a TEM specimen with a large thin area, we may use all these steps except dimpling. Dimpling is helpful to make a TEM specimen quickly, but the specimen thin area will be limited. To obtain a high quality TEM specimen, every step needs to be controlled well. Some key steps and their effects on specimen microstructures will be discussed.

Besides sample materials, the goals of TEM experiments are a key factor to choose specimen preparation method and parameters. In general, the modified conventional method works well for diffraction contrast imaging and tripod-related method is useful for high resolution (S)TEM imaging.

[1] The samples were provided by Rong Zhang, Philippe Spiberg and Manijeh Razeghi. 

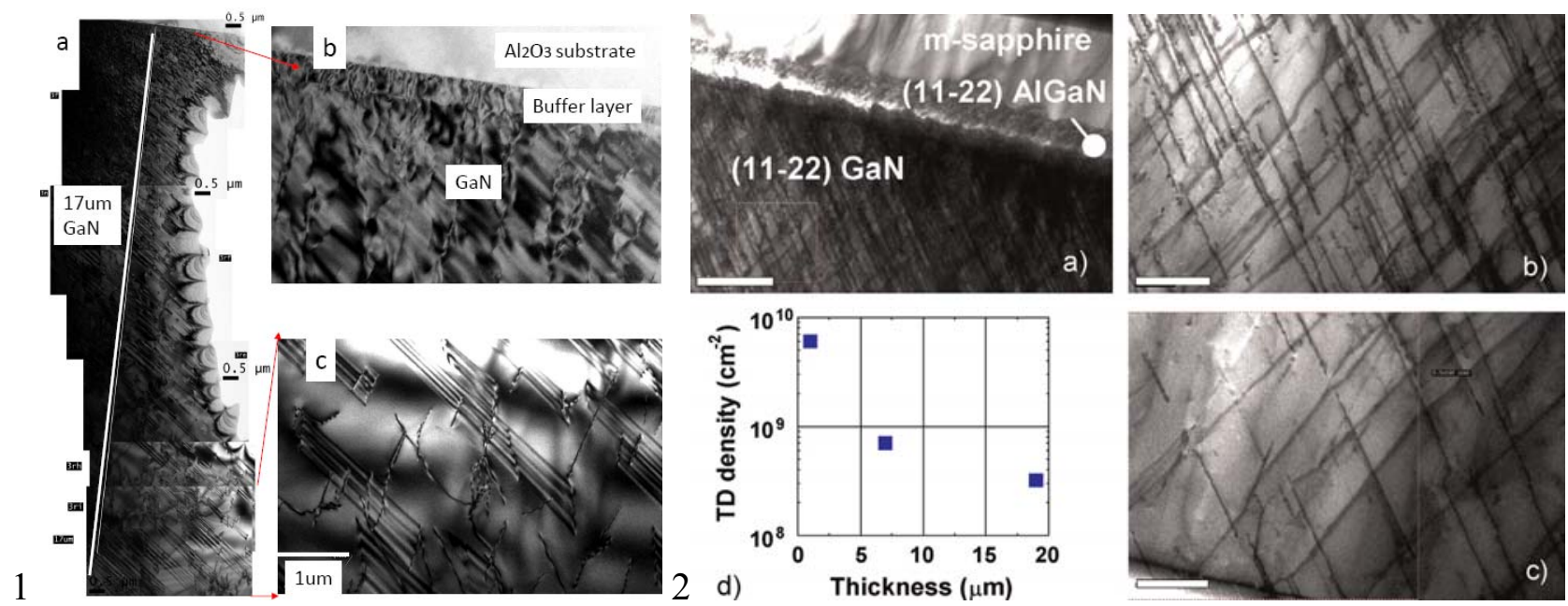

Fig. 1 a) $17 \mu \mathrm{m}$ thick polar GaN layer grown on (0001) sapphire substrate, where defects can be imaged from GaN top surface to the substrate, b) Thread dislocations (TDs) in GaN layer in the area close to the substrate. c) Stacking faults and TDs close to the GaN top surface.

Fig. 2 Thread dislocations (TDs) in a $20 \mu \mathrm{m}$ thick semi-polar GaN layer grown on m-plane sapphire substrate in TEM images recorded a) close to the substrate, b) about $7 \mu \mathrm{m}$ from the substrate and c) about $20 \mu \mathrm{m}$ from the substrate or close to the GaN surface. The scale bar is $1 \mu \mathrm{m}$. d) the decreasing of the TD density with layer thickness.
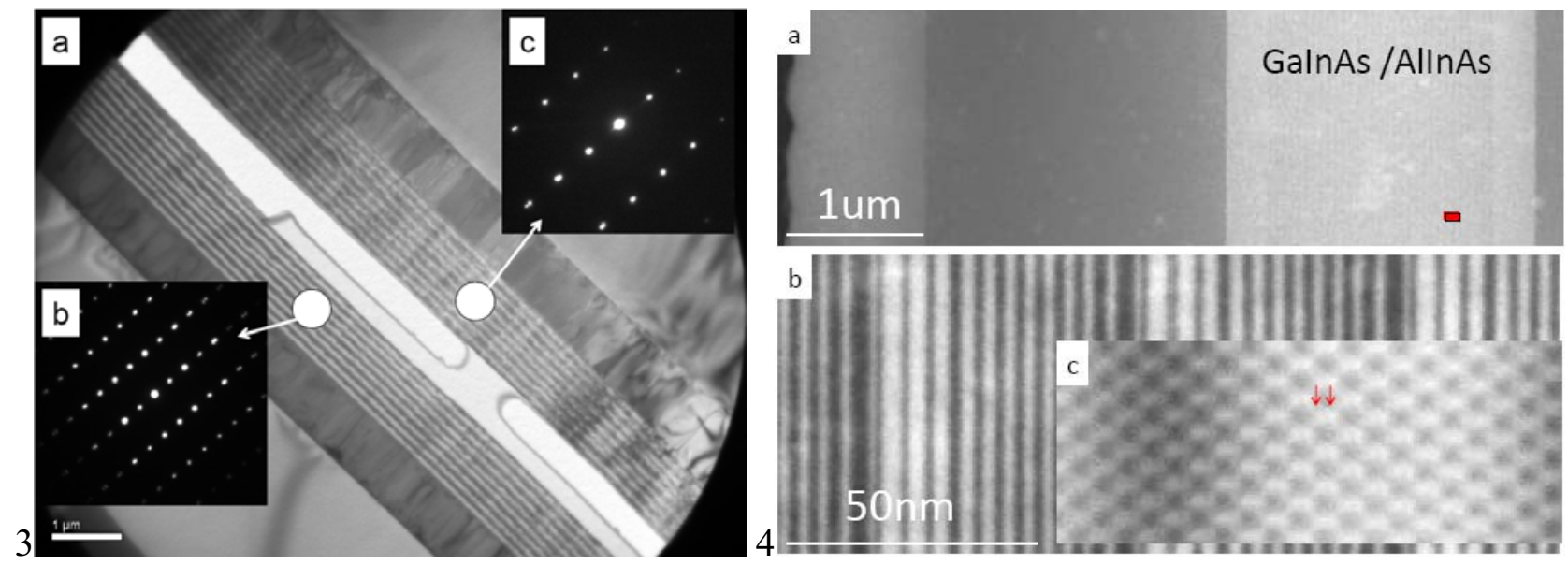

Fig. 3 a) GaN/AlN superlattice grown on GaN buffer and sapphire substrate and prepared by mechanical polishing and ion milling. Two pieces of the superlattice were glued together with 30 degree relative rotation. The glue line thickness is less than $1 \mu \mathrm{m} . \mathrm{b}$ ) and c) are selected area electron diffraction patterns recorded from the superlattice areas showing a 30 degree relative rotation.

Fig. 4 a) Cross-sectional HAADF STEM image of a semiconductor device. The specimen was prepared by a tripod-related polishing method. b) Part of GaInAs/AlInAs superlattice from the red bar area marked in a), c) High resolution Z-contrast STEM image of the coherent interface between GaInAs (bright area) and AlInAs (dark area) at the atomic scale. The dumb-bell atomic columns are indicated by the pair of red arrows. 